Robert C. Powers and Thomas Riedel, Department of Mathematics, University of Louisville, Louisville, KY 40292, USA,

e-mail: rcpowe01@@homer.louisville.edu \& t0ried01@@homer.louisville.edu

\title{
WHEN ARE CONTINUOUS ISOTONE BIJECTIONS ORDER AUTOMORPHISMS?
}

\begin{abstract}
The purpose of this paper is to study weakly continuous isotone bijections on a lattice of residuated maps (e.g. the space of probability distribution functions). We investigate the connection between weakly continuous isotone bijections and order automorphisms. Our final result gives necessary and sufficient conditions for a continuous isotone bijection to be an order automorphism.
\end{abstract}

\section{Introduction}

In this paper we study weakly continuous bijections on lattices of residuated maps. We employ certain lattice theoretic methods which were developed by the first author in $[6,7]$. In these papers order automorphisms and certain residuated mappings on such lattices were studied and it was shown that these mappings essentially act by left and right composition. This representation for order automorphisms was used to show that they are weakly continuous functions. We are guided by the comparison of these mappings with monotone functions on real intervals, and we show how the lack of a total order has some interesting consequences, by giving an example of a class of weakly continuous isotone bijections whose inverses are not order preserving. Next, we briefly drop the assumption of continuity and prove some facts about isotone bijections. Our main result, Theorem 3.3, is a description of all the continuous bijections that are, in fact, order automorphisms. This leads to Corollary 3.8,

Key Words: isotone bijection, order automorphism, residuated map, weakly continuous Mathematical Reviews subject classification: Primary: 06A23, 06B30, 54C35

Received by the editors July 8, 1994 
our final result, where we give necessary and sufficient conditions for a weakly continuous isotone bijection to be an order automorphism. We conclude with some remarks and open problems.

This paper is divided into three sections, Section 1 being this introduction. In Section 2 we introduce the relevant notation and some preliminary results, and Section 3 contains our main results.

\section{Preliminaries}

In this section we introduce some necessary notation and basic results to keep this paper reasonably self-contained. For details we refer the reader to $[1,2$, $6,7]$.

A mapping between two complete lattices is residuated (respectively, resid$u a l$ ) if it preserves arbitrary suprema (respectively, arbitrary infima). For example, if $[p, q]$ and $[r, s]$ are (possibly extended) nondegenerate real intervals then $\phi:[p, q] \rightarrow[r, s]$ is residuated if $\phi$ is monotone nondecreasing, leftcontinuous and $\phi(p)=r$. We let $\Delta^{*}$ denote the set of all residuated mappings from $[p, q]$ into $[r, s]$. One possibility for $\Delta^{*}$ is the set of all left-continuous, cumulative probability distribution functions of non-negative random variables, see $[4,8]$. Under the usual pointwise partial ordering of functions,

$$
\phi_{1} \leq \phi_{2} \text { in } \Delta^{*} \Longleftrightarrow \phi_{1}(x) \leq \phi_{2}(x) \text {, for all } x \in[p, q]
$$

$\Delta^{*}$ is a complete lattice, i.e., every subset of $\Delta^{*}$ has a supremum and infimum in $\Delta^{*}$.

For readers more familiar with lattice theory, we point out that $\Delta^{*}$ is actually a completely distributive lattice and therefore a continuous lattice (for more information see $[1,3]$ ). Many of the questions raised here can be studied in this more general context.

Here we are, however, interested in the specific case of $\Delta^{*}$ and it's applications; furthermore, the methods used in this paper (see $[6,7]$ ) were developed for this particular case.

For each $\phi$ in $\Delta^{*}$ there is an associated residual map $\phi^{+}:[r, s] \rightarrow[p, q]$ given by

$$
\phi^{+}(y)=\sup \{x \in[p, q] \mid \phi(x) \leq y\} .
$$

Note that if $x \in[p, q]$ and $y \in[r, s]$ then $\phi(x) \leq y$ if and only if $x \leq \phi^{+}(y)$. This means that $\phi^{+}(y)$ is actually the maximum of $\{x \in[p, q] \mid \phi(x) \leq y\}$. Also, for each $\phi$ in $\Delta^{*}, l^{+} \phi(p)$ denotes the right hand limit of $\phi$ at $p$, that is

$$
l^{+} \phi(p)=\inf \{\phi(t) \mid t>p\}
$$


A mapping between two complete lattices is an order isomorphism if it is an isotone (order-preserving) bijection whose inverse is also isotone; it is a dual order isomorphism if it is an antitone (order-reversing) bijection whose inverse is also antitone. An order isomorphism from a complete lattice onto itself is called an order automorphism.

The following theorem characterizing the order automorphisms of $\Delta^{*}$ was proven in $[6]$.

Theorem 2.1 (Characterization Theorem) The mapping $\Gamma$ is an order automorphism of $\Delta^{*}$ if and only if one of the following holds:

(i) there are order automorphisms $\theta$ of $[r, s]$ and $\tau$ of $[p, q]$, such that $\Gamma(\phi)=$ $\theta \circ \phi \circ \tau$ for all $\phi$ in $\Delta^{*}$;

(ii) there are dual order isomorphisms $\alpha$ and $\beta$ from $[p, q]$ onto $[r, s]$ such that $\Gamma(\phi)=\alpha \circ \phi^{+} \circ \beta$, for all $\phi \in \Delta^{*}$.

The following elements of $\Delta^{*}$ are of particular interest and therefore deserve special symbols. Let $a \in[p, q], b \in[r, s]$, then

$$
\delta_{a, b}(x)= \begin{cases}r, & \text { if } x \leq a \\ b, & \text { if } x>a\end{cases}
$$

and

$$
\mu_{a, b}(x)= \begin{cases}r, & \text { if } x=p \\ b, & \text { if } p<x \leq a \\ s, & \text { if } x>a\end{cases}
$$

We note that $\delta_{p, r}$ and $\delta_{p, s}$ are, respectively the least and greatest elements of $\Delta^{*}$ and that $\delta_{a, s}=\mu_{a, r}$ for all $a \in[p, q]$, and $\delta_{p, b}=\mu_{q, b}$ for all $b \in[r, s]$. Furthermore, in lattice-theoretic terms, the $\delta_{a, b}$ 's are the join irreducible and the $\mu_{a, b}$ 's are the meet irreducible elements in the lattice $\Delta^{*}$.

We will also need the following topological properties of $\Delta^{*}$, which can be found in [8]. A sequence $\left\{\phi_{n}\right\}_{n=1}^{\infty}$ in $\Delta^{*}$ is said to converge weakly to $\phi$ in $\Delta^{*}$, in symbols $\phi_{n} \stackrel{w}{\rightarrow} \phi$, if $\lim _{n \rightarrow \infty} \phi_{n}(x)=\phi(x)$ for every $x$ in the set of continuity points of $\phi$ (see [5] and [9]). We say a mapping $\Gamma: \Delta^{*} \rightarrow \Delta^{*}$ is weakly continuous if $\phi_{n} \stackrel{w}{\rightarrow} \phi$ implies that $\Gamma\left(\phi_{n}\right) \stackrel{w}{\rightarrow} \Gamma(\phi)$. If it is clear from the context, we will omit the word weakly.

\section{Continuous Isotone Bijections}

If $\Delta^{*}$ is the set of all left-continuous cumulative distribution functions of nonnegative random variables then there exists a metric $d_{L}^{*}$ on $\Delta^{*}$ such that

$$
d_{L}^{*}\left(\phi_{n}, \phi\right) \rightarrow 0 \text { if and only if } \phi_{n} \stackrel{w}{\rightarrow} \phi
$$


In this case $[p, q]=[0, \infty]$ and $[r, s]=[0,1]$ and $d_{L}^{*}$ is the modified Lévy metric defined by

$$
d_{L}^{*}(\phi, \psi)=\inf \{h \in(0,1] \mid \text { both }[\phi, \psi ; h] \text { and }[\psi, \phi ; h] \text { hold }\}
$$

where $[\phi, \psi ; h]$ denotes the condition $\psi(x) \leq \phi(x+h)+h$ for all $x \in\left(0, \frac{1}{h}\right)$. Furthermore, the metric space $\left(\Delta^{*}, d_{L}^{*}\right)$ is compact (see $\left.[8,10]\right)$.

Since any two nondegenerate closed subintervals of the extended reals are order isomorphic, it follows that for any possible $\Delta^{*}$ there is a metric $d$ satisfying equation (3.1), such that $\left(\Delta^{*}, d\right)$ is compact. Therefore, if $\Gamma: \Delta^{*} \rightarrow \Delta^{*}$ is a continuous isotone bijection then it's inverse is necessarily continuous, i.e., $\Gamma$ is a homeomorphism. The next example, however, shows that the inverse of a continuous isotone bijection of $\Delta^{*}$ need not be isotone. In this example, for the sake of clarity, we let $[p, q]=[r, s]=[0,1]$.

Example 3.1 Let $\Delta^{*}$ be the set of all left-continuous isotone $\phi:[0,1] \longrightarrow$ $[0,1]$. Then there exists $a \Gamma: \Delta^{*} \longrightarrow \Delta^{*}$ such that $\Gamma$ is a continuous isotone bijection whose inverse is not isotone.

To show this let $d:[0,1] \longrightarrow[0,1]$ be given by $d(x)=1-x$, then $d$ is a dual order automorphism of $[0,1]$. Further, for any $\phi \in \Delta^{*}$ we let $t=\sup \{x \in$ $[0,1] \mid \phi(x) \leq d(x)\}$, (note that $t$ is just the $x$-coordinate of the point at which the graph of $\phi$ intersects or jumps over the graph of $1-x)$ and define $\theta_{\phi}:[0,1] \rightarrow[0,1]$ by

$$
\theta_{\phi}(x)= \begin{cases}x, & \text { if } x \leq 1-t \\ \frac{2}{3} x+\frac{1}{3}-\frac{t}{3}, & \text { if } 1-t<x \leq \frac{4-t}{4}, \\ 2 x-1, & \text { if } \frac{4-t}{4}<x\end{cases}
$$

Thus $\theta_{\phi}$ is a piecewise linear function belonging to Aut $[0,1]$.

We define $\Gamma: \Delta^{*} \rightarrow \Delta^{*}$ by $\Gamma(\phi)=\theta_{\phi} \circ \phi$ for each $\phi \in \Delta^{*}$. It is straightforward to verify that $\Gamma$ is isotone. To show continuity, let $\phi_{n}$ converge weakly to $\phi$ in $\Delta^{*}$, let $t_{n}=\sup \left\{x \in[0,1] \mid \phi_{n}(x) \leq 1-x\right\}$ and $t=\sup \{x \in[0,1] \mid \phi(x) \leq 1-x\}$, then it is easily seen that $\lim _{n \rightarrow \infty} t_{n}=t$. Thus $\theta_{\phi_{n}}$ converges pointwise to $\theta_{\phi}$, and therefore $\Gamma\left(\phi_{n}\right)$ converges weakly to $\Gamma(\phi)$. Next we observe that $\Gamma$ is a bijection with inverse $\Gamma^{-1}(\phi)=\theta_{\phi}^{-1} \circ \phi$.

To show that the inverse of $\Gamma$ does not preserve order, we first show how $\Gamma$ acts on the $\delta_{a, b}$ 's. To this end we note that

$$
t=\sup \left\{x \in[0,1] \mid \delta_{a, b}(x) \leq 1-x\right\}= \begin{cases}1-b & \text { if } b \leq 1-a, \\ a & \text { if } 1-a<b .\end{cases}
$$


Thus, we have

$$
\Gamma\left(\delta_{a, b}\right)= \begin{cases}\delta_{a, b} & \text { if } b \leq 1-a \\ \delta_{a, \frac{2 b}{3}+\frac{1}{3}-\frac{a}{3}} & \text { if } 1-a<b \leq \frac{4-a}{4}, \\ \delta_{a, 2 b-1} & \text { if } \frac{4-a}{4} \leq b .\end{cases}
$$

Now consider $\delta_{\frac{1}{2}}, \frac{1}{2}$ and $\delta_{\frac{2}{3}}, \frac{7}{12}$, which are non-comparable. However,

$$
\Gamma\left(\delta_{\frac{2}{3}, \frac{7}{12}}\right)=\delta_{\frac{2}{3}, \frac{1}{2}} \leq \delta_{\frac{1}{2}, \frac{1}{2}}=\Gamma\left(\delta_{\frac{1}{2}, \frac{1}{2}}\right),
$$

and hence $\Gamma^{-1}$ does not preserve order, whence $\Gamma$ is not an order automorphism.

There are many different variations of the example given above: use arbitrary intervals $[p, q],[r, s]$; change the dual order isomorphism $d$; change the order automorphism $\theta_{\phi}$; or compose $\Gamma$ with one or more continuous isotone bijections of $\Delta^{*}$. Moreover, we could modify Example 3.1 to obtain an isotone bijection which is not continuous. In the future we plan to investigate these variations and, hopefully, give an order theoretic characterization of all such examples.

This example shows that an isotone bijection on $\Delta^{*}$, even a continuous one, need not be an order automorphism. This is unlike the case for bijections on real intervals, where every isotone bijection is continuous, hence an order automorphism. Thus we proceed to investigate some properties of isotone bijections on $\Delta^{*}$.

Theorem 3.2 Let $\Gamma: \Delta^{*} \rightarrow \Delta^{*}$ be an isotone bijection, and let $\phi$ be in $\Delta^{*}$.

(i) If $\phi^{+}(r)>p$ and $\phi(q)<s$ then $\Gamma(\phi)^{+}(r)>p$ and $\Gamma(\phi)(q)<s$.

(ii) If $l^{+} \phi(p)>r$ and $\phi(t)=s$ for some $t<q$ then $l^{+} \Gamma(\phi)(p)>r$ and $\Gamma(\phi)\left(t^{\prime}\right)=s$ for some $t^{\prime}<q$.

The proof of Theorem 3.2 uses the following observations. If $\Gamma: \Delta^{*} \rightarrow \Delta^{*}$ is isotone and $\Gamma\left(\phi_{1}\right)$ incomparable to $\Gamma\left(\phi_{2}\right)$, then $\phi_{1}$ is incomparable to $\phi_{2}$. Furthermore, if $\phi$ is incomparable to $\delta_{a, b}$, then $\phi(a) \leq b$ and hence $\phi \leq \mu_{a, b}$. Finally, if $\phi$ is incomparable to $\mu_{a, b}$ then $\phi(a)>b$ and so $\phi \geq \delta_{a, b}$.

Proof. (i) Suppose $\phi^{+}(r)>p$ and $\phi(q)<s$, then $\phi \leq \delta_{\phi^{+}(r), \phi(q)}=\delta_{a, b}$, where $a>p$ and $b<s$. Assume that $\Gamma(\phi)^{+}(r)=p$, then, since $\Gamma(\phi) \leq \Gamma\left(\delta_{a, b}\right)$, it follows that $\Gamma\left(\delta_{a, b}\right)^{+}(r)=p$. Now let $\left\{\phi_{n}\right\}$ be a sequence in $\Delta^{*}$ such that $\Gamma\left(\phi_{n}\right)=\delta_{p+1 / n, s}$, for $n$ large enough that $p+1 / n \in[p, q]$. Note that either $\phi_{n}$ is incomparable to $\delta_{a, b}$ or $\phi_{n} \leq \delta_{a, b}$. In either case we get $\phi_{n} \leq \mu_{a, b}$ for every $n$. Thus $\Gamma\left(\phi_{n}\right) \leq \Gamma\left(\mu_{a, b}\right)$ for every $n$. Since $\Gamma\left(\phi_{n}\right)=\delta_{p+1 / n, s}$, we have that $\delta_{p+1 / n, s} \leq \Gamma\left(\mu_{a, b}\right)<\delta_{p, s}$ for all $n$. This leads to a contradiction, since $\sup _{n} \delta_{p+1 / n, s}=\delta_{p, s}$. Thus $\Gamma(\phi)^{+}(r)>p$. 
Assume now that $\Gamma(\phi)(q)=s$. Then $\Gamma\left(\delta_{a, b}\right)(q)=s$. Let $\left\{\tau_{n}\right\}$ be a sequence in $\Delta^{*}$ satisfying $\Gamma\left(\tau_{n}\right)=\delta_{p, s-1 / n}$. Since $\Gamma\left(\delta_{a, b}\right)^{+}(r)>p$ and $\Gamma\left(\delta_{a, b}\right)(q)=s$ it follows that $\Gamma\left(\tau_{n}\right)$ is incomparable to $\Gamma\left(\delta_{a, b}\right)$ for every $n$. Therefore $\tau_{n} \leq \mu_{a, b}$ and so $\delta_{p, s-1 / n}=\Gamma\left(\tau_{n}\right) \leq \Gamma\left(\mu_{a, b}\right)$ for every $n$. But $\Gamma\left(\mu_{a, b}\right)<\delta_{p, s}$, hence we have again a contradiction and thus $\Gamma(\phi)(q)<s$.

(ii) Let $\phi \in \Delta^{*}$ satisfy $l^{+} \phi(p)>r$ and $\phi(t)=s$ for some $t<q$. Further let $a=\inf \{x \in[p, q] \mid \phi(x)=s\}$ and $b=l^{+} \phi(p)$. Then $a<q, b>r$ and $\phi \geq \mu_{a, b}$. Now assume that $l^{+} \Gamma(\phi)(p)=r$. Then $l^{+} \Gamma\left(\mu_{a, b}\right)(p)=r$. Let $\left\{\phi_{n}\right\} \subset \Delta^{*}$ be such that $\Gamma\left(\phi_{n}\right)=\delta_{p, r+1 / n}$, then either $\Gamma\left(\phi_{n}\right) \geq \Gamma\left(\mu_{a, b}\right)$ or $\Gamma\left(\phi_{n}\right)$ is incomparable to $\Gamma\left(\mu_{a, b}\right)$. Hence either $\phi_{n} \geq \mu_{a, b}$ or $\phi_{n}$ is incomparable to $\mu_{a, b}$. In either case $\phi_{n} \geq \delta_{a, b}$, hence $\delta_{p, r+1 / n}=\Gamma\left(\phi_{n}\right) \geq \Gamma\left(\delta_{a, b}\right)>\delta_{p, r}$ for all $n$. This contradiction yields $l^{+} \Gamma(\phi)(p)>r$.

For the second part assume that $\Gamma(\phi)(x)<s$ for all $x<q$. In this case $\Gamma\left(\mu_{a, b}\right)(x)<s$ for all $x<q$. Now let $\left\{\tau_{n}\right\} \subset \Delta^{*}$ be the sequence satisfying $\Gamma\left(\tau_{n}\right)=\delta_{q-1 / n, s}$. Since $l^{+} \Gamma\left(\mu_{a, b}\right)(p)>r$ and $\Gamma\left(\mu_{a, b}\right)(x)<s$ for every $x<q$, it follows that $\Gamma\left(\tau_{n}\right)$ is incomparable to $\Gamma\left(\mu_{a, b}\right)$, and thus $\tau_{n}$ is incomparable to $\mu_{a, b}$ for every $n$. This in turn implies that $\tau_{n} \geq \delta_{a, b}$ for every $n$, hence $\delta_{q-1 / n, s}=\Gamma\left(\tau_{n}\right) \geq \Gamma\left(\delta_{a, b}\right)>\delta_{q, s}$, which is a contradiction. Thus $\Gamma(\phi)\left(t^{\prime}\right)=s$ for some $t^{\prime}<q$.

If we define $J^{\prime}=\left\{\phi \in \Delta^{*} \mid \phi^{+}(r)>p\right.$ and $\left.\phi(q)<s\right\}$ and $M^{\prime}=\left\{\phi \in \Delta^{*} \mid\right.$ $l^{+} \phi(p)>r$ and $\phi(t)=s$ for some $\left.t<q\right\}$, then Theorem 3.2 states that every isotone bijection on $\Delta^{*}$ maps $J^{\prime}$ into $J^{\prime}$ and $M^{\prime}$ into $M^{\prime}$. This is analogous to the fact that every order automorphism maps $J=\left\{\delta_{a, b} \mid a \in[p, q)\right.$ and $b \in$ $(r, s]\}$ into $J$ and $M=\left\{\mu_{a, b} \mid a \in[p, q)\right.$ and $\left.b \in(r, s]\right\}$ into $M$. Note that while $M \subset M^{\prime}, J$ and $J^{\prime}$ have a large intersection, neither one is contained in the other. In fact we have $J \backslash\left\{\delta_{a, s} \mid a \in[p, q)\right\} \subset J^{\prime}$.

Having stated the properties of isotone bijections on $\Delta^{*}$, we now proceed to answer the question posed in our title. To this end we explore the role of continuity, again guided by the result for bijections on real intervals. In the real case, any bijection which is continuous and isotone on a set containing at least two elements is an order automorphism. Recall from Example 3.1 that this is not the case on $\Delta^{*}$. This leads us to a third condition, requiring that $J$ and $M$ be mapped into themselves in a prescribed way.

Let $F=\left\{\phi \in \Delta^{*} \mid\right.$ the range of $\phi$ is finite subset of $\left.[r, s]\right\}$. So each element in $F$ is a step function with a finite number of steps. It follows that $J$ and $M$ are subsets of $F$. A function $\Gamma: \Delta^{*} \rightarrow \Delta^{*}$ is isotone on $F$ if $\phi_{1} \leq \phi_{2}$ in $F$ implies $\Gamma\left(\phi_{1}\right) \leq \Gamma\left(\phi_{2}\right)$ in $\Delta^{*}$, i.e., $\left.\Gamma\right|_{F}$ is isotone.

Theorem 3.3 Let $\Gamma: \Delta^{*} \rightarrow \Delta^{*}$ be a bijection. Then $\Gamma$ is an order automorphism of $\Delta^{*}$ if and only if the following three conditions hold: 
(i) $\Gamma$ is continuous;

(ii) $\Gamma$ is isotone on $F$;

(iii) for each $a$ in $(p, q)$ and $b$ in $(r, s)$,

$$
\Gamma\left(\delta_{a, b}\right)=\delta_{x, y} \text { and } \Gamma\left(\mu_{a, b}\right)=\mu_{x, y}
$$

for some $x$ in $[p, q]$ and $y$ in $[r, s]$

If $\Gamma$ is an order automorphism of $\Delta^{*}$, then it follows from [6], the definition of an order automorphism and the Characterization Theorem that $\Gamma$ satisfies (i), (ii) and (iii), respectively. Thus it remains to show that any bijection of $\Delta^{*}$ satisfying (i), (ii) and (iii) is actually an order automorphism.

Instead of showing directly that the inverse is isotone, we give a constructive proof that involves a series of lemmas. For example, in our next result, we show that a continuous bijection satisfying equation (3.2) fixes the smallest and largest elements of the lattice $\Delta^{*}$. In addition, such a bijection maps the chain $V_{p}=\left\{\delta_{p, b} \mid b \in[r, s]\right\}$ into itself or into the chain $H_{s}=\left\{\delta_{a, s} \mid a \in[p, q]\right\}$. Notice that these facts hold without assuming that the bijection is isotone.

Lemma 3.4 Let $\Gamma: \Delta^{*} \rightarrow \Delta^{*}$ be a bijection satisfying conditions (i) and (iii) in Theorem 3.3. Then

(a) $\Gamma\left(\delta_{p, r}\right)=\delta_{p, r}$ and $\Gamma\left(\delta_{p, s}\right)=\delta_{p, s}$.

(b) Either $\Gamma\left(V_{p}\right)$ is a subset of $V_{p}$ or of $H_{s}$.

(c) Either $\Gamma\left(H_{s}\right)$ is a subset of $H_{s}$ or of $V_{p}$.

Proof. (a) We will show that $\Gamma\left(\delta_{p, s}\right)=\delta_{p, s}$. Let $\{p+1 / n\},\{s-1 / n\}$ be sequences in $(p, q)$ and $(r, s)$, respectively. Then $\delta_{p+1 / n, s-1 / n} \stackrel{w}{\rightarrow} \delta_{p, s}$ and $\mu_{p+1 / n, s-1 / n} \stackrel{w}{\rightarrow} \mu_{p, s}=\delta_{p, s}$. By equation (3.2) we have

$$
\Gamma\left(\delta_{p+1 / n, s-1 / n}\right)=\delta_{x_{n}, y_{n}} \text { and } \Gamma\left(\mu_{p+1 / n, s-1 / n}\right)=\mu_{x_{n}, y_{n}} .
$$

By working with subsequences when necessary, we can assume that the sequences $\left\{x_{n}\right\}$ and $\left\{y_{n}\right\}$ converge to some points $x \in[p, q]$ and $y \in[r, s]$, respectively. It follows from the continuity of $\Gamma$ that $\Gamma\left(\delta_{p, s}\right)=\delta_{x, y}$ and $\Gamma\left(\delta_{p, s}\right)=\Gamma\left(\mu_{p, s}\right)=\mu_{x, y}$. Since $\delta_{x, y}=\mu_{x, y}$ it follows that either $x=p$ and $y=s$ or $x=q$ and $y=r$, i.e., either $\Gamma\left(\delta_{p, s}\right)=\delta_{p, s}$ or $\Gamma\left(\delta_{p, s}\right)=\delta_{q, r}=\delta_{p, r}$.

To eliminate the second possibility, let $b \in(r, s)$ such that $\Gamma\left(\delta_{p, b}\right) \neq \delta_{p, r}$. Let $\{p+1 / n\}$ be a sequence in $(p, q)$. By equation (3.2), we have

$$
\Gamma\left(\delta_{p+1 / n, b}\right)=\delta_{u_{n}, v_{n}} \text { and } \Gamma\left(\mu_{p+1 / n, b}\right)=\mu_{u_{n}, v_{n}}
$$

where $u_{n} \in[p, q]$ and $v_{n} \in[r, s]$ for each $n$. Using the same argument as above, we have that $\Gamma\left(\delta_{p, b}\right)=\delta_{u, v}$ and $\Gamma\left(\mu_{p, b}\right)=\mu_{u, v}$, for some $u \in[p, q]$ and 
$v \in[r, s]$. Note that $\mu_{p, b}=\delta_{p, s}$. Since $\Gamma\left(\delta_{p, b}\right) \neq \delta_{p, r}$ it follows that $u \neq q$ and $v \neq r$. Hence $\mu_{u, v} \neq \delta_{p, r}$ and so $\Gamma\left(\delta_{p, s}\right)=\delta_{p, s}$.

A similar argument shows that $\Gamma\left(\delta_{p, r}\right)=\delta_{p, r}$.

(b) Let $\delta_{p, b} \in V_{p}$ such that $b \in(r, s)$. Following the argument in (a) we get $\Gamma\left(\delta_{p, b}\right)=\delta_{x, y}$ and $\Gamma\left(\mu_{p, b}\right)=\mu_{x, y}$ for some $x \in[p, q]$ and $y \in[r, s]$. Since $\mu_{p, b}=\delta_{p, s}$, it follows from (a) that either $x=p$ or $y=s$, which gives us $\Gamma\left(V_{p} \cup H_{s}\right) \subset V_{p} \cup H_{s}$.

Let $A=\left\{b \in(r, s) \mid \Gamma\left(\delta_{p, b}\right) \in V_{p}\right\}$ and let $B=\left\{b \in(r, s) \mid \Gamma\left(\delta_{p, b}\right) \in H_{s}\right\}$. By (a) and by the fact that $\Gamma$ is one-to-one, the sets $A$ and $B$ are disjoint. By the first paragraph, $A \cup B=(r, s)$. Next, suppose $\left\{b_{i}\right\}$ is a sequence in $A$ with limit point $b$ in $(r, s)$. Then $b \in A$. To see this, note that $\Gamma\left(\delta_{p, b_{i}}\right)=\delta_{p, c_{i}}$ for each $i$ and $\lim _{i \rightarrow \infty} \delta_{p, b_{i}}=\delta_{p, b}$. By continuity, $\Gamma\left(\delta_{p, b}\right)=\lim _{i \rightarrow \infty} \delta_{p, c_{i}}$ and from the above either $\Gamma\left(\delta_{p, b}\right) \in V_{p}$ or $\Gamma\left(\delta_{p, b}\right) \in H_{s}$. It follows that $\Gamma\left(\delta_{p, b}\right) \in V_{p}$, i.e., $b \in A$. Thus $A$ is closed in $(r, s)$. Similarly, $B$ is closed in $(r, s)$. Since $(r, s)$ is an interval either $A$ or $B$ is empty. Thus either $\Gamma\left(V_{p}\right)$ is a subset of $V_{p}$ or $\Gamma\left(V_{p}\right)$ is a subset of $H_{s}$.

A similar argument establishes the result for $\Gamma\left(H_{s}\right)$.

The next result is straightforward.

Lemma 3.5 The set $F$ is a dense subset of $\Delta^{*}$.

In Lemmas 3.6 and 3.7 we obtain the two types of order automorphisms of $\Delta^{*}$, described by (i) and (ii), respectively, in the Characterization Theorem.

Lemma 3.6 Let $\Gamma: \Delta^{*} \rightarrow \Delta^{*}$ be a bijection that satisfies conditions (i), (ii) and (iii) in Theorem 3.3. If $\Gamma\left(V_{p}\right)$ is a subset of $V_{p}$, then for each $\phi \in \Delta^{*}$, $\Gamma(\phi)=\theta \circ \phi \circ \tau$, where $\theta$ is an order automorphism of $[r, s]$ and $\tau$ is an order automorphism of $[p, q]$.

Proof. Since $\Gamma\left(V_{p}\right) \subset V_{p}$, we can define a function $\theta:[r, s] \rightarrow[r, s]$ via $\theta(b)=y$ whenever $\Gamma\left(\delta_{p, b}\right)=\delta_{p, y}$. Note that we have $\theta(r)=r$ and $\theta(s)=s$. Furthermore, $\theta$ is continuous, order-preserving and one-to-one since $\Gamma$ is weakly continuous, isotone and one-to-one. The fact that $\theta$ is also onto follows from the intermediate value property of continuous functions on real intervals. Thus $\theta$ is an order automorphism of $[r, s]$ and $\Gamma\left(V_{p}\right)=V_{p}$.

Since $\Gamma$ is also one-to-one it follows from Lemma 3.4 that $\Gamma\left(H_{s}\right) \subset H_{s}$. Now we can define a function $\sigma:[p, q] \rightarrow[p, q]$, via $\sigma(a)=x$ whenever $\Gamma\left(\delta_{a, s}\right)=\delta_{x, s}$. It follows, as in the above, that $\sigma$ is an order automorphism of $[p, q]$.

Let $a \in(p, q)$ and $b \in(r, s)$, then, using equation (3.2) it follows that $\Gamma\left(\delta_{a, b}\right)=\delta_{x, y}$ and $\Gamma\left(\mu_{a, b}\right)=\mu_{x, y}$ for some $x \in[p, q]$ and some $y \in[r, s]$. Since 
$\Gamma$ is isotone on $F$ and $\delta_{a, b} \leq \delta_{a, s} \leq \mu_{a, b}$ we have $\delta_{x, y} \leq \delta_{\sigma(a), s} \leq \mu_{x, y}$ and hence $x=\sigma(a)$. Similarly it follows that $y=\theta(b)$. Thus $\Gamma\left(\delta_{a, b}\right)=\delta_{\sigma(a), \theta(b)}=$ $\theta \circ \delta_{a, b} \circ \sigma^{-1}$, and $\Gamma\left(\mu_{a, b}\right)=\mu_{\sigma(a), \theta(b)}=\theta \circ \mu_{a, b} \circ \sigma^{-1}$, for all $a \in[p, q]$ and $b \in[r, s]$. We have thus shown that $\Gamma$ acts on the two sets $J$ and $M$ by left and right composition with $\theta$ and $\sigma^{-1}$, respectively. It remains to prove that this extends to all of $\Delta^{*}$.

To this end let $\phi \in F$, for $x \in(p, q]$ let $a=\sigma^{-1}(x)$ and $b=\phi(a)$. Now we define a sequence of elements of $\Delta^{*}$ as follows: Let $a_{n}=\sigma^{-1}(x-1 / n)$ and $b_{n}=\phi\left(a_{n}\right)$, where $n$ is large enough such that $x-1 / n \in[p, q]$, then $\delta_{a_{n}, b_{n}}=\delta_{a_{n}, \phi\left(a_{n}\right)} \leq \phi \leq \mu_{a, b}$. Since $\Gamma$ is isotone on $F$ we have

$$
\theta\left(b_{n}\right)=\delta_{x-1 / n, \theta\left(b_{n}\right)}(x) \leq \Gamma(\phi)(x) \leq \mu_{x, \theta(b)}(x)=\theta(b) .
$$

Since $\lim _{n \rightarrow \infty} \theta\left(b_{n}\right)=\theta(b)$ we have that $\Gamma(\phi)(x)=\theta(b)=\theta\left(\phi\left(\sigma^{-1}(x)\right)\right)$ for any $x \in[p, q]$. Thus $\Gamma(\phi)=\theta \circ \phi \circ \tau$, where $\tau=\sigma^{-1}$, for any $\phi \in F$. This extends to all of $\Delta^{*}$ by applying Lemma 3.5.

Lemma 3.7 Let $\Gamma: \Delta^{*} \rightarrow \Delta^{*}$ be a bijection satisfying conditions (i), (ii) and (iii) in Theorem 3.3. If $\Gamma\left(V_{p}\right)$ is a subset of $H_{s}$, then for each $\phi \in \Delta^{*}$, $\Gamma(\phi)=\alpha \circ \phi^{+} \circ \beta$ where $\alpha, \beta$ are dual order isomorphisms from $[p, q]$ onto $[r, s]$.

Proof. Since $\Gamma\left(V_{p}\right) \subset H_{s}$, we can define a function $\varepsilon:[r, s] \rightarrow[p, q]$ via $\varepsilon(b)=$ $x$ whenever $\Gamma\left(\delta_{p, b}\right)=\delta_{x, s}$. It is easy to verify that $\varepsilon$ is a dual order isomorphism and thus $\Gamma\left(V_{p}\right)=H_{s}$. This implies, using Lemma 3.4 that $\Gamma\left(H_{s}\right) \subset V_{p}$, and we can define $\alpha:[r, s] \rightarrow[p, q]$ by $\alpha(a)=y$ whenever $\Gamma\left(\delta_{a, s}\right)=\delta_{p, y}$.

Following the proof of the above lemma, it is easily verified that $\Gamma\left(\delta_{a, b}\right)=$ $\delta_{\varepsilon(b), \alpha(a)}=\alpha \circ \delta_{a, b} \circ \varepsilon^{-1}$ and $\Gamma\left(\mu_{a, b}\right)=\mu_{\varepsilon(b), \alpha(a)}=\alpha \circ \mu_{a, b} \circ \varepsilon^{-1}$ for all $a \in[p, q]$ and all $b \in[r, s]$.

Thus it remains to show that $\Gamma$ acts in the same way on all of $\Delta^{*}$. We proceed as in the previous lemma. Let $\phi \in F$, for $x \in(p, q]$ we let $a=$ $\phi^{+}\left(\varepsilon^{-1}(x)\right)$, and note that, since $\phi \leq \mu_{a, \phi(a)}$ we have $\Gamma(\phi) \leq \Gamma\left(\mu_{a, \phi(a)}\right)=$

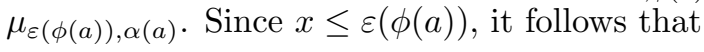

$$
\Gamma(\phi)(x) \leq \alpha(a)=\left(\alpha \circ \phi^{+} \circ \varepsilon^{-1}\right)(x) .
$$

Furthermore, since $\phi(y) \leq t$ if and only if $y \leq \phi^{+}(t)$, we have for every $t \in[r, s]$, where $n$ is large enough such that $x-1 / n \in[p, q]$ that $\delta_{\phi^{+}(t), t} \leq \phi$. Now let $t_{n}=\varepsilon^{-1}(x-1 / n)$, then it follows from $\delta_{\phi^{+}\left(t_{n}\right), t_{n}} \leq \phi$ that

$$
\Gamma\left(\delta_{\phi^{+}\left(t_{n}\right), t_{n}}\right)=\delta_{x-1 / n, \alpha\left(\phi^{+}\left(t_{n}\right)\right)} \leq \Gamma(\phi) .
$$


Therefore we have $\delta_{x-1 / n, \alpha\left(\phi^{+}\left(t_{n}\right)\right)}(x)=\alpha\left(\phi^{+}\left(t_{n}\right)\right) \leq \Gamma(\phi)(x)$ for every $n$. This yields $\alpha\left(\phi^{+}\left(\varepsilon^{-1}(x-1 / n)\right)\right) \leq \Gamma(\phi)(x)$, and by left-continuity,

$$
\alpha\left(\phi^{+}\left(\varepsilon^{-1}(x)\right)\right) \leq \Gamma(\phi)(x) \text {, for every } x \in[p, q] .
$$

Thus we have that $\Gamma(\phi)=\alpha \circ \phi^{+} \circ \varepsilon^{-1}$ for every $\phi \in F$, and by letting $\beta=\varepsilon^{-1}$, this yields the result on $F$. This equation extends to all of $\Delta^{*}$ by applying Lemma 3.5.

Notice that Lemmas 3.4 through 3.7 complete the proof of Theorem 3.3. The next result is an immediate consequence of this theorem, and gives an answer to the question posed in our title.

Corollary 3.8 Let $\Gamma: \Delta^{*} \rightarrow \Delta^{*}$ be a continuous isotone bijection. Then $\Gamma$ is an order automorphism of $\Delta^{*}$ if and only if, for each a in $(p, q)$ and $b$ in $(r, s)$,

$$
\Gamma\left(\delta_{a, b}\right)=\delta_{x, y} \text { and } \Gamma\left(\mu_{a, b}\right)=\mu_{x, y}
$$

for some $x$ in $[p, q]$ and $y$ in $[r, s]$.

Notice that Example 3.1 demonstrates that condition (iii) in Theorem 3.3 is necessary. Now if we let $[p, q]=[0,1]=[r, s]$, it is easy to verify that the function $\Gamma: \Delta^{*} \rightarrow \Delta^{*}$ given by $\Gamma(\phi)=\phi$ if $\phi \in F$ and $\Gamma(\phi)=(\phi)^{2}$ if $\phi \in \Delta^{*}-F$ is a bijection that satisfies conditions (ii) and (iii) in Theorem 3.3 but not condition (i). Thus condition (i) is also necessary. Finally, we do not know if condition (ii) in Theorem 3.3 is necessary.

In closing we note that the authors have been made aware of the fact that the result of Corollary 3.8 may be true for continuous isotone bijections on more general lattices. Preliminary investigations showed that since the Characterization Theorem was proved only for the case of $\Delta^{*}$, a completely different proof is necessary. This question is currently under investigation by the authors.

\section{References}

[1] G. Birkhoff, Lattice Theory, Amer. Math. Soc., Providence, 3rd. edition, 1967.

[2] T. S. Blyth and M. F. Janowitz, Residuation Theory, Pergamon Press, London, 1972.

[3] G. Gierz, K. H. Hofmann, J. D. Keimel, K. Lawson, M. Mislove, and D. S. Scott, A Compendium of Continuous Lattices, Springer-Verlag, BerlinHeidelberg-New York, 1980. 
[4] M. F. Janowitz and B. Schweizer, Ordinal and percentile clustering, Math. Social Sciences, 18 (1989), 135-186.

[5] M. Loève, Probability Theory, Springer-Verlag, New York, 1977.

[6] R. C. Powers, Order automorphisms of spaces of nondecreasing functions, J. Math. Anal. Applic., 136 (1988), 112-123.

[7] R. C. Powers, Induced residuated mappings, Order, 7 (1991), 83-96.

[8] B. Schweizer and A. Sklar, Probabilistic Metric Spaces, North-Holland, New York, 1983.

[9] D. A. Sibley, A Metric for weak convergence of distribution functions, Rocky Mountain J. of Math., 1 (1972), 427-430.

[10] R. M. Tardiff, Topologies for probabilistic metric spaces, $\mathrm{PhD}$ thesis, Univ. of Massachusetts, Amherst, 1975. 